\title{
Development of IAEA nuclear reaction databases and services
}

\author{
V. Zerkin ${ }^{1, \mathrm{a}}$ and A. Trkov ${ }^{1, \mathrm{~b}}$ \\ Department of Nuclear Sciences and Applications, International Atomic Energy Agency, 1400 Vienna, Austria
}

\begin{abstract}
From mid-2004 onwards, the major nuclear reaction databases (EXFOR, CINDA and ENDF) and services (Web and CD-ROMs retrieval systems and specialized applications) have been functioning within a modern computing environment as multiplatform software, working under several operating systems with relational databases. Subsequent work at the IAEA has focused on three areas of development: revision and extension of the contents of the databases; extension and improvement of the functionality and integrity of the retrieval systems; development of software for database maintenance and system deployment.
\end{abstract}

\section{Introduction}

EXFOR (experimental data), CINDA (bibliographic information) and ENDF (recommended evaluated data) are major nuclear reaction databases that are available via Web services [1], CD-ROM retrieval systems and specialised applications. Extensive work began in 1999 on the migration of the major nuclear data databases and services from VMS to a modern computer environment [2,3]. This initiative has been successfully completed through the co-operative efforts of the IAEA Nuclear Data Section (IAEA-NDS) and the National Nuclear Data Center (NNDC), Brookhaven National Laboratory - the new system was released by both centres in mid-2004 [4]. Subsequent to this initiative, mirror-sites of the IAEA-NDS Web system were installed and are successfully functioning in two regional Nuclear Data Centers: BARC (India) and IPEN (Brazil).

Since mid-2004 further development of the system at the IAEA has focused on three areas: revision and extension of the contents of the databases; extension and improvement of the functionality and integrity of the retrieval systems; development of software for database maintenance, system deployment and extensions by user's applications.

\section{Revision and extension of the contents of the database}

The IAEA nuclear data services now offer access to improved and extended nuclear reaction databases:

- a common NRDC EXFOR library available since 2005 , implemented via IAEA-NDS software for comparison of full EXFOR libraries of four nuclear data centres;

- a new CINDA library extended to include references for charged-particle and photonuclear measurements; new

\footnotetext{
${ }^{a}$ Presenting author, e-mail: V.Zerkin@iaea.org

${ }^{b}$ Present address: Jozef Stefan Institute, 1000 Ljubljana, Slovenia
}

software to import missing information from EXFOR to CINDA was developed and used regularly for automatic extension of the CINDA database;

- ENDF database extended by data libraries that represent the products of various IAEA projects and other sources (currently 27 libraries); this extended database will be used as a comprehensive repository for all evaluated libraries (including specialized and archival old versions) to ensure that the data is kept available for the retrieval system, including data search, presentation in various forms and plotting.

\section{Extension and improvement of the retrieval systems}

Major improvements of the IAEA nuclear reaction retrieval systems include the following features:

- extension of plotting capabilities (e.g., figs. 1-3);

- inter-database links;

- new output formats;

- flexible ENDF database explorer.

\subsection{Extension of plotting capabilities}

The plotting capabilities were extended in the following ways:

- plot of data describing angular distributions, doubledifferential distributions, energy spectra and cross sections with errors of evaluated data (MF33 involved);

- simplified output format suitable for import to other plotting software;

- interactive Web plotting with immediate effect: zoom by mouse, actions by one click, more functions (grid, autorange, title, etc.), similar to ZVView functionality;

- possibility to add user's data (experimental and curves) to plot on Web.

These extended plotting features are implemented in EXFORENDF Web retrieval system and EndVer/GUI CD-ROM 


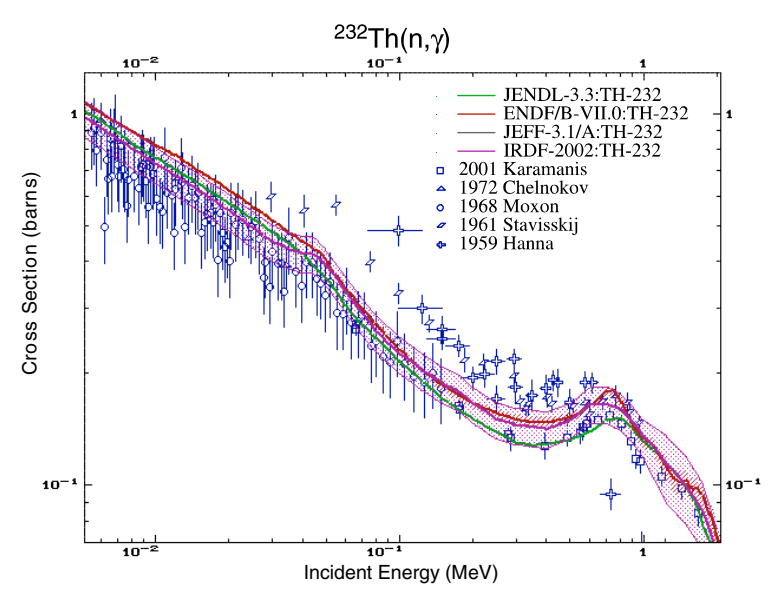

Fig. 1. Cross sections with errors of evaluated data.

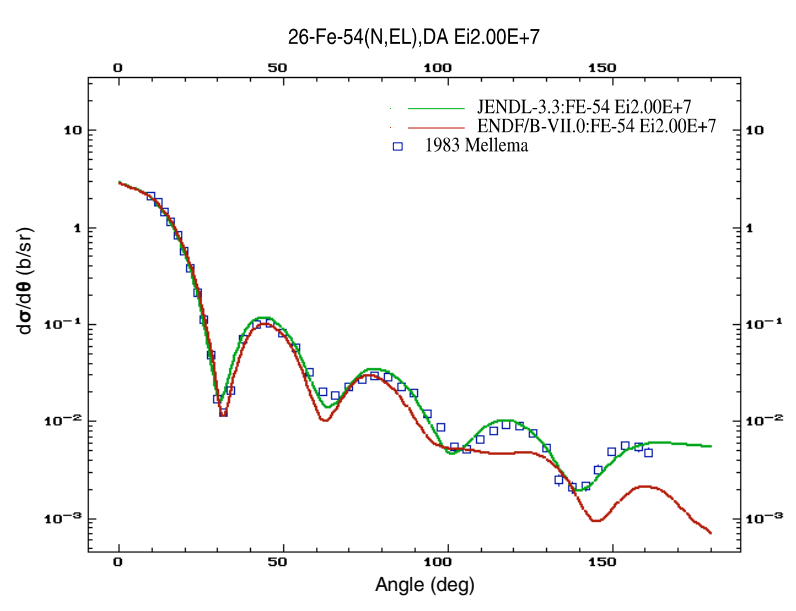

Fig. 2. Angular distributions.

combining database search, EndVer utilities [5], PrePro [6] and X4toC4 codes with ZVView [7] plotting program.

\subsection{Inter-database links}

Web interface to all three CINDA, EXFOR, ENDF databases allows any request for data to be copied to each other (i.e., make "clones" of the request). The system also offers:

- CINDA-EXFOR links following explicit reference in CINDA;

- CINDA-NSR and EXFOR-NSR: during loading, data programs find corresponding references in the NSR database to be used "on the fly" calling the NSR Web retrieval system;

- EXFOR and CINDA link to Web-Journals by reference (abstract, full article), using information from NSR and the internal Dictionary;

- EXFOR-ENDF: search similar data and plot them all.

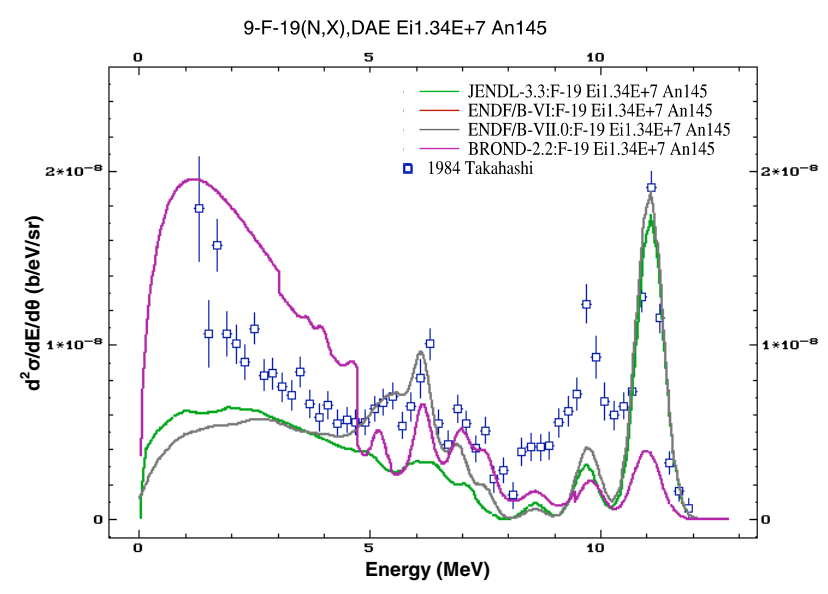

Fig. 3. Double-differential cross sections.

\subsection{New output formats}

Several new output data types have been introduced for adoption by the user's software: BibTeX and EXFOR+ (EXFOR extended by information from dictionaries, explicitly giving volume, pages, journal name of the reference, better readable data, etc.); table formats for EXFOR and ENDF cross sections (simple column format with minimum identifying information).

\subsection{Flexible ENDF database explorer}

The EXFOR-CINDA-ENDF retrieval system was released in 2004, and was designed primarily for the users with having well-defined tasks involving data search, downloading and presentation. The system offers "direct" types of request with any combination of search parameters. The advantage of this method is that users, who know exactly what they need, can access the data quickly and without any limits; disadvantage is that users have no a priori information about the existence of the data they require nor a knowledge of the full contents of the database, (also - identification of "similar" data needs significant experience). A natural solution to these disadvantages is a "sequential" type of search where the user adopts a multi-step procedure of the data search, obtaining only possible values of the parameters on every step of the request (system ensures that user acquires the existing data).

An example of such a retrieval system is the old NNDC ENDF set-up on the VMS (Web and Telnet) [8]. Modern implementations of the sequential search use tree representations of the data which are commonly used in file-managers of many operating systems (e.g., MS Windows-Explorer) and in nuclear data packages (Janis [9]) that offer to view and search data by opening/closing nodes of the tree. The normal limitation of the sequential approach involves the fixed nature of the chain of properties to be "opened" (e.g., Target-Reaction-Quantity-Library-Data). A "flexible database explorer" allows the user to select the sequence of properties on the fly and provides an additional service. At the present time, this concept has only been implemented for ENDF database on the Web. 
The tasks for the development of the flexible ENDF database explorer have been were defined as follows:

- implement sequential data search without fixing the chains of properties to be opened;

- provide a compact summary report of the database contents at any step and for every property;

- allow integral data selection at every step, limited only by the time of data retrieval and the volume of data to be transferred;

- provide full traditional services after the data have been selected.

At every step, the user can open a next level by choosing a "switch" corresponding to one of the properties or requesting a summary report for the same property: target (cascade: element - isotope), reaction (cascade: projectile - reaction), quantity, library. Data for further retrieval and standard service can be selected by clicking on "folder" or "paper" (fig. 4).

The system has several additional useful features and options:

- few fixed chains of properties;

- quick selection of target material using periodic table;

- extended summary reports (e.g., full list of materials of the given library);

- view types (large, medium, small).

\section{Development of software tools}

The main task in the development of system tools is to provide and simplify the maintenance, access and distribution of the databases minimizing programming efforts.

\subsection{Universal programs}

Several universal programs were developed for many purposes (most of these programs are platform-independent):

- universal GUI (Graphical Users Interface) - Java program for database control and maintenance, observation of the file system and running other programs and scripts, etc. The program is used to load and update all three database, dictionary database, main user's interface in EndVer/GUI CD-ROM and other applications;

- program for loading XML data to the database (loading of original data to a database is done in two steps: production of the file in simplified XML format, and loading of this file to a database);

- SQL commands execution;

- auto-configuration system: Web and CD-ROM programs detect their location and the environment in which they are running. All the programs are configured "on the fly" (for example avoids installation of the Web retrieval systems on the IAEA mirror Web sites). CD-ROM systems can run from $\mathrm{CD}$ without being copied to a hard drive.

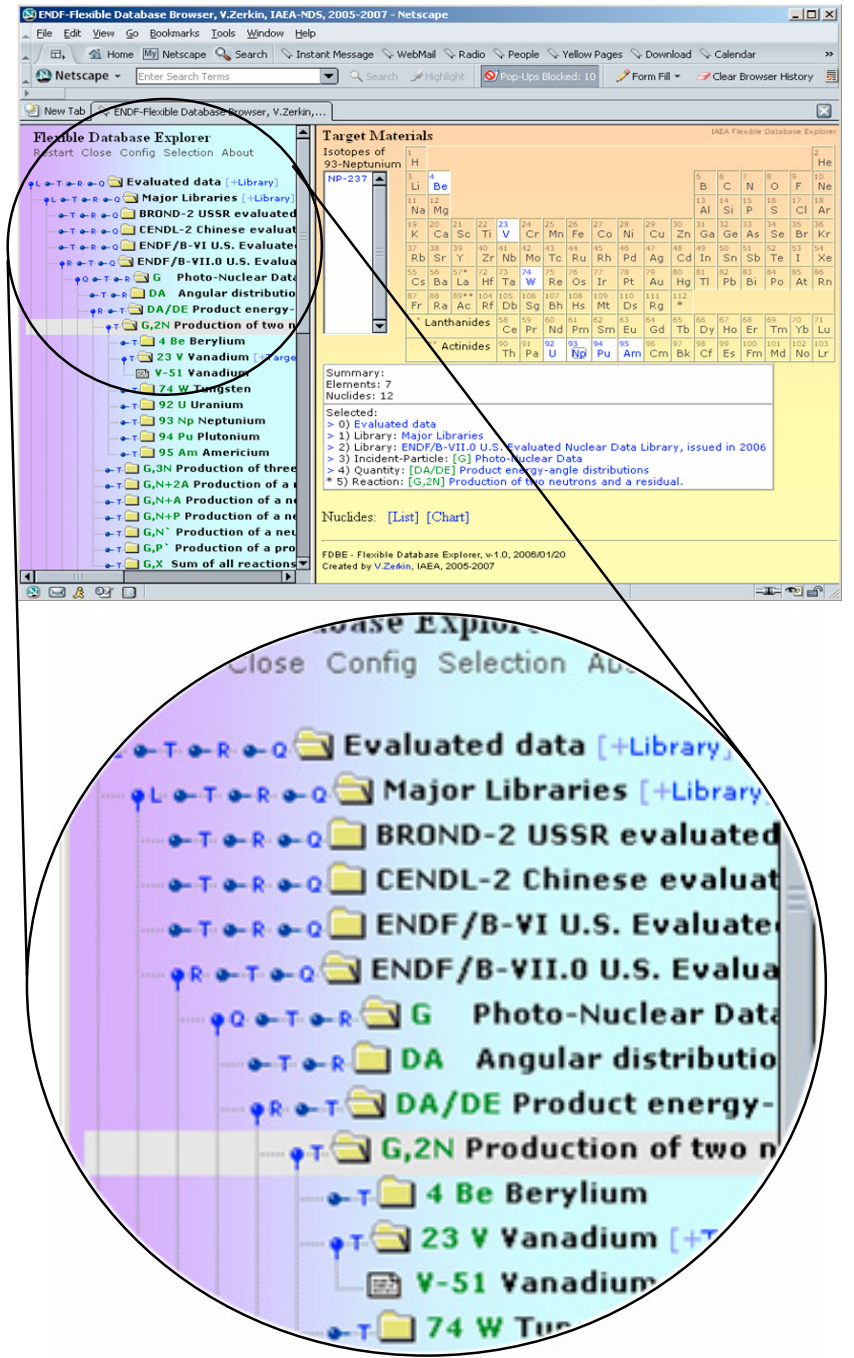

Fig. 4. Flexible ENDF database explorer.

\subsection{EXFOR-CINDA tools}

The CINDA database has been extended by photonuclear and charged particle reaction data (work undertaken in cooperation with other nuclear data centres). Apart from the standard software maintaining the CINDA database and retrieval systems, CINDA tools also include:

- software for automatic extension of the database by importing new information from EXFOR;

- CINDA editor;

- backup system.

EXFOR tool includes:

- platform-independent checking code used in the compilation process (inherited from McLane, NNDC, ported to Linux/Windows and extended regularly);

- dictionary database maintenance software;

- backup system;

- non-interactive retrieval program. 


\section{Advanced access to the data and services}

All extended and improved databases and services are accessible via Web retrieval systems on IAEA-NDS and mirror sites and on CD-ROMs.

As well as the traditional features of CD-ROMs with database and retrieval system [10], the products on the CD are foreseen to undergo extended usage:

- database files (MS-Access and MySQL) can be used for specific queries and for building new retrieval systems [11];

- prepared to be used as part of a user's application.

CD-ROM "EXFOR-CINDA for Applications" works on Linux and MS-Windows, and has full EXFOR and CINDA databases as MySQL with interactive and non-interactive retrieval systems. All software is pre-configured and prepared to work as a stand-alone system or through being called from another package. This approach allows access to the full database from an external application without having to study the SQL and database structure. The package is successfully used in EndVer/GUI CD-ROM and in the EMPIRE package [12].

CD-ROM "EndVer/GUI" is built on top of CD "EXFORCINDA for Applications", and integrates the ENDF file verification package with full EXFOR database. The package allows the retrieval and plotting of the experimental and evaluated cross-section data (including differential and doubledifferential data). PrePro utilities, X4toC4 code and ZVView interactive plotting program are built-in. Using configuration files, the package can be extended by the user's software.

\section{Conclusions}

Tasks described above are performed by implementation of extensive new software that has extended and improved both the contents of the databases and quality of the services. The resulting system is more comprehensive, sophisticated and user-friendly, and provides convenient tools for data compilations and database management. Web services can easily be cloned on the IAEA mirror sites to create rapid and efficient regional network services. Systems on a CD-ROM can be used as local services and as a data source for advanced applications.

\section{References}

1. IAEA Nuclear Data Services: http://www-nds.iaea.org/.

2. V. Pronyaev, D. Winchell, V. Zerkin, D. Muir, R. Arcilla, Requirements for the Next Generation of Nuclear Databases and Services, pp. 1476-1479, in J. Nucl. Sci. Technol., Suppl. 2, Proc. Int. Conf. on Nuclear Data for Science and Technology, Tsukuba, Japan, 7-12 October 2001.

3. V.V. Zerkin, V. McLane, M.W. Herman, C.L. Dunford, EXFORCINDA-ENDF: Migration of Databases to give Higher Quality Nuclear Data Services, in Proc. Int. Conf. on Nuclear Data for Science and Technology, Santa Fe, New Mexico, USA, 26 Sep. - 1 Oct. 2004, pp. 586-589, AIP conference proceedings 769, edited by R.C. Haight, M.B. Chadwick, T. Kawano, P. Talou (College Park, MD., American Institute of Physics, 2005).

4. B. Pritychenko, A.A. Sonzogni, D.F. Winchell, V.V. Zerkin, R. Arcilla, T.W. Burrows, C.L. Dunford, M.W. Herman, V. McLane, P. Oblozinsky, Y. Sanborn, J.K. Tuli, Nuclear reaction and structure data services of the National Nuclear Data Center, Ann. Nucl. Energy 33, 390 (2006).

5. A. Trkov, ENDVER-ENDF File verification support package, http://www-nds.iaea.org/ndspub/endf/endver/.

6. D.E. Cullen, ENFB Pre-processing Codes Pre-Pro-96, IAEANDS-39, Vienna, Austria, 1996.

7. V. Zerkin, ZVView - graphics software for nuclear data analysis, http://www-nds.iaea.org/ndspub/zvview/.

8. C.L. Dunford, T.W. Burrows, Online nuclear data service, IAEA-NDS-150, Rev. 99/3, Vienna, Austria, 1999.

9. A. Nouri, P. Nagel, N. Soppera, A. Ahite, B. Taton, J. Patrouix, F. Lecompagnon, C. Cunin, O. Rioland, L. d'Eurveilher, JANIS: A New Software for Nuclear Data Services, in J. Nucl. Sci. Technol., Suppl. 2, Proc. Int. Conf. on Nuclear Data for Science and Technology, Tsukuba, Japan, 7-12 October 2001, pp. 14801483.

10. V. Zerkin, Stand-alone retrieval system on CD-ROM: EXFOR + CINDA/Java2, in Report on IAEA Consultants' Meeting on the Co-ordination of Nuclear Reaction Data Centres (Technical Aspects), 17-19 June 2003, INDC(NDS)-446, WP2003-24, pp. 144-146, Vienna, Austria, 2003.

11. Russian Nuclear data Centre (Centr Jadernykh Dannykh-CJD), http://www.ippe.obninsk.ru/podr/cjd/page4_9_cjd.html.

12. M. Herman, R. Capote, B. Carlson, P. Obložinsky, M. Sin, A. Trkov, V. Zerkin, EMPIRE nuclear reaction model code, Version 2.19 (Lodi), http://www.nndc.bnl.gov/empire219/ (2005). 